screening, which can be used by commissioners to evaluate and plan their local service provision. The model and an analysis of national data have been recently published in BMJ. Increasingly, financial constraints will mean that tools such as ours will be vital to provide value for money in healthcare.

Method We will give a live demonstration of our spreadsheet model using the example from our recent study. We invite delegates to input their own data in real time to a laptop for comparison and testing against the national and international picture. We plan to promote this before the meeting.

Main outcome measures Cost effectiveness of screening (as cost per individual tested, cost per positive diagnosis, total cost of screening, number screened, number infected, sex ratio of those tested and treated). We will show how to compare current programmes with a planned change eg, in screening coverage or partner notification efficacy. Results In 2008-2009 screening was estimated to cost $£ 46.3 \mathrm{~m}$ and $£ 506$ per infection treated. The model results suggest that increasing male screening coverage from $8 \%$ to $24 \%$ (to match female coverage) would cost an extra $£ 22.9 \mathrm{~m}$ and increase the cost per infection treated to £528. Increasing partner notification efficacy from 0.4 to 0.8 partners per index case would cost an extra $£ 3.3 \mathrm{~m}$ and reduce the cost per infection diagnosed to $£ 449$.

Conclusions The results are broadly representative of the national picture, but further evaluation of the cost effectiveness of partner notification and screening is urgently needed. We encourage practitioners who want to test this tool in their own setting to contact us (katy.turner@bristol.ac.uk).BMJ 2011; 342:c7250 10.1136/bmj.c7250.

\section{P1-S4.18 USING MATHEMATICAL MODELLING TO INVESTIGATE THE ROLE OF THE HIDDEN “POPULATION OF MEN WHO HAVE SEX WITH MEN (MSM) ON THE HIV EPIDEMIC IN SOUTHERN INDIA"}

doi:10.1136/sextrans-2011-050108.162

${ }^{1} \mathrm{H}$ Prudden, ${ }^{1} \mathrm{~A}$ Foss, ${ }^{1} \mathrm{~K}$ Mitchell, ${ }^{2} \mathrm{M}$ Pickles, ${ }^{2} \mathrm{~A}$ Phillips, ${ }^{3,4} \mathrm{~B} \mathrm{M}$ Ramesh, ${ }^{3,4,5} \mathrm{R}$ Washington, ${ }^{6} \mathrm{M}$ Alary, ${ }^{7} \mathrm{C}$ Lowndes, ${ }^{1} \mathrm{P}$ Vickerman. ${ }^{1}$ London School of Hygiene and Tropical Medicine, London, UK; ${ }^{2}$ Imperial College London, London, UK; ${ }^{3}$ Karnataka Health Promotion Trust, Bangalore, India and; " University of Manitoba Winnipeg, Canada; ${ }^{5}$ St John's Research Institute, Bangalore, India; ${ }^{6}$ Centre hospitalier affilié universitaire de Québec, Québec, Canada; ' Health Protection Agency, London, UK Canada

Background Biological and behavioural data for men who have sex with men (MSM) in Bangalore, Karnataka, India, have mainly been collected from sites where commercial sex is prevalent. Consequently, the survey data may fail to capture the behaviour of a larger lower-risk "hidden" MSM population. Mathematical modelling is used to explore the potential bias in the survey data and better quantify the characteristics of this hidden population.

Methods A dynamic model of HIV transmission among MSM was developed and parameterised using detailed data* from high risk MSM in urban Bangalore. The MSM were categorised into three subgroups: Kothi and Hijra(KH): who mostly take the receptive role in anal sex, Panthis and Bisexuals(PB) who are predominantly insertive partners and Double Deckers(DD) who take both roles. Due to the sampling methods used, it was thought the MSM survey data were more representative of $\mathrm{KH}$ and $\mathrm{DD}$ than $\mathrm{PB}$, although the extent of this bias is unknown. Therefore, no fitting constraint was applied to the PB HIV prevalence and instead the model was used to explore what PB HIV prevalence values are projected if the model was only fit to the $95 \%$ CIs of the prevalence data for $\mathrm{KH}$ and DD. One million randomly sampled model simulations were undertaken to find model fits.

Results Abstract P1-S4.18 figure 1 shows that, although the model can produce HIV prevalence estimates consistent with the survey estimates, overall the model projections suggest a lower PB HIV prevalence is more consistent with the survey estimates for $\mathrm{KH}$ and DD. In addition, $80 \%$ of the model fits to the $\mathrm{KH}$ and DD HIV prevalence data had a sampled frequency of sex acts for $\mathrm{PB}$ in the lower half of the uncertainty interval suggesting PB's sexual activity may be lower than the median reported in the MSM survey. As expected, an inverse relationship occurs between $\mathrm{PB}$ population size and their level of sexual activity, with the median $\mathrm{PB}$ population size being 55400 (2.7\% of the total urban male population) and varying between 28000 and 73000 (1.5 to 3.9\% of the total urban male population) for the IOR of the model fits Conclusions: Survey data imply MSM are a small, highly active group, many of whom regularly sell sex, have very high numbers of partners and typically take the receptive role. As demonstrated here, modelling can be used to provide insights into the likely HIV prevalence, population size and sexual activity of hidden "MSM not reached in surveys."

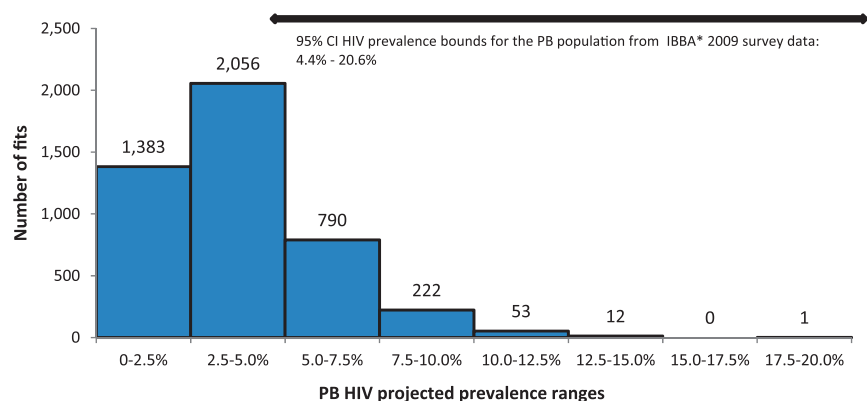

Abstract P1-S4.18 Figure 1 Number and range of projected HIV prevalence estimates for the PB population (from the model fits to $\mathrm{KH}$ and DD data. * Integratedbiological and behavioural assessment (IBBA) 2009 , collected within the monitoring and evaluation of Avahan, the India AIDS initiative.

\section{P1-S4.19 STOCHASTIC NETWORK MODELS}

doi:10.1136/sextrans-2011-050108.163

P A Noël, A Allard, L Hébert-Dufresne, V Marceau, L J. Dubé. Université Laval, Québec, Canada

Background We present a general modelling scheme particularly adapted for the epidemiology of sexually transmitted infections. Emphasis is placed on the complex interaction structure of the population and on the probabilistic nature of the dynamics.

Methods We represent the interaction structure of the population with complex network models. Markov Stochastic Processes are used to consider the probabilistic time evolution of both the network structure and the epidemiological state of the population. Idealised epidemiological problems are considered: they are qualitatively inspired by real-world systems but no actual real-world data is fed to the models. All analytical results are systematically validated through Monte Carlo numerical simulations.

Results By dedicating compartments to individuals with both similar epidemiological states and similar contact patterns, we observe great agreement between analytical results and Monte Carlo simulations see Abstract P1-S4.19 Figure 1. This is a special case of the general observation that compartmental models perform better when individuals within each compartment are very similar among themselves. Explicit examples are given for contact patterns changing through time as well as for the interactions of different infections in the same population.

Conclusions By specifying the structure of the population through its local features, we successfully model the probabilistic evolution of complex epidemiological systems. Infection stages and/or behavioural groups are considered the same way they are in "classical" compartmental models. The generality of the approach facilitates its application to a vast array of epidemiological phenomena. 


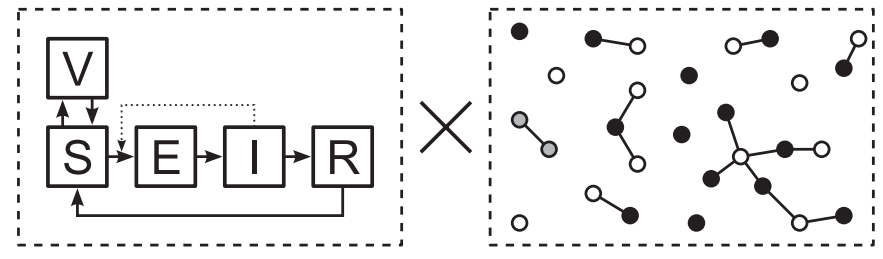

Abstract P1-S4.19 Figure 1 Epidemiology on networks.

\section{P1-S4.20 MATHEMATICAL MODELLING OF HIV TRANSMISSION AND CONTROL AMONG MEN WHO HAVE SEX WITH MEN: A REVIEW OF 25 YEARS OF LITERATURE}

doi:10.1136/sextrans-2011-050108.164

${ }^{1} \mathrm{~N}$ Punyacharoensin, ${ }^{1}$ W J Edmunds, ${ }^{2} \mathrm{D}$ De Angelis, ${ }^{1} \mathrm{R}$ Guy White. ${ }^{1}$ London School of Hygiene \& Tropical Medicine, London, UK; ${ }^{2}$ Heath Protection Agency and MRC Biostatistics Unit, UK

Background For a quarter of century, mathematical models have been used to study the spread and control of HIV among men who have sex with men (MSM). We reviewed this literature to summarise the methodologies used, key model developments, and recommended strategies for HIV control among MSM.

Methods Review of the literature on dynamic compartmental models of HIV transmission among MSM was conducted. MEDLINE/EMBASE were searched from earliest date to end 2010.

Results Of 742 studies identified, 127 studies met the inclusion criteria and were selected for review. Most studies employed deterministic methods (80\%), and potentially as a result of this, as the complexity of models increased over time with respect to antiretroviral therapy (ART), there was a marked decline in the complexity of models with respect to sexual activity. Only a small proportion of models were fitted to data (22\%) and even fewer were validated $(17 \%)$, somewhat reducing confidence in the findings from these studies. That said, a number of common findings emerged, including (1) the importance of assumed changes in infectivity and sexual contact rates on the impact of ART on HIV incidence, and that this led to follow-up empirical studies to gather these data, and (2) the recommendation that multiple strategies would be required for effective HIV control among MSM.

Conclusions Mathematical models have been useful in indentifying key empirical studies and for showing that multiple prevention strategies would be required for effective control of HIV epidemics in MSM. The lack of model fitting and validation emphasise that this area should be targeted for developments in the future. An improved methodology for parameter estimation will help generate predictions that more fully express uncertainty, allowing more informed public heath decision making.

\section{P1-S4.21 CALIBRATION OF AN INDIVIDUAL-BASED MODEL OF STI TRANSMISSION IN UGANDA: A NOVEL ABC-BAYESIAN EMULATION HYBRID APPROACH}

doi:10.1136/sextrans-2011-050108.165

H Johnson, W John Edmunds, R White. London School of Hygiene and Tropical Medicine, London, UK

Introduction The complexity of dynamic epidemic systems has led to the use of large-scale stochastic models for prediction purposes. However, methods for robustly calibrating and analysing these models can be prohibitively inefficient. We propose an algorithm for fitting complex models that incorporates elements of both Approximate Bayesian Computation $(\mathrm{ABC})$ and Bayesian Emulation. $\mathrm{ABC}$ enables inference about model parameters without needing to calculate a likelihood function, by generating approx- imations from repeated model runs. However, each model run might take hours. Emulation methods are being developed in the fields of cosmology and meteorological modelling. The complex model function is summarised as an "emulator": a stochastic function that represents global behaviour of the function as a linear regression model and local deviations from this behaviour as Gaussian processes. The emulator acts as a cheap proxy for the complex model, allowing both calibration and sensitivity analysis to be conducted in a fraction of the time.

Methods We report the initial application of an emulation-based calibration algorithm to an individual-based stochastic model of STI transmission in Uganda. Starting with uninformative priors for 19 behavioural and biological input parameters, we "trained" an emulator with 200 sampled parameter sets and their corresponding model output (point estimates of HIV prevalence). Sampling a further 10000 parameter sets from the priors, we used the emulator to make output predictions over a large area of input parameter space. Weighting each parameter set by goodness of fit to observed data, we identified promising areas of parameter space for complex model evaluation. A more accurate emulator was then trained, incorporating this additional complex model output. The process was repeated as in sequential $A B C$ methods.

Results The use of emulators allowed evaluation of large areas of parameter space due to increased computational efficiency. Processing time for one prevalence point estimate was reduced from over $15 \mathrm{~min}$ on an HPC cluster to less than $0.1 \mathrm{~s}$ on a PC. Even the first two waves of such an algorithm provided helpful insight into the most influential parameters

Conclusions The development of an ABC-Bayesian Emulation hybrid approach to complex model calibration is promising. Emulators offer large advantages in computational efficiency. However, further research is needed regarding weighting, tolerance levels and covariance.

\section{P1-S4.22 IMPACT OF A HYPOTHETICAL CHLAMYDIA VACCINE ON POPULATION PREVALENCE: A MATHEMATICAL MODELLING STUDY}

doi:10.1136/sextrans-2011-050108.166

${ }^{1} \mathrm{~J}$ Heijne, ${ }^{1} \mathrm{C}$ Althaus, ${ }^{1} \mathrm{~S}$ Herzog, ${ }^{1} \mathrm{~N}$ Low. ${ }^{1}$ University of Bern, Bern, Switzerland

Background It has not yet been possible to develop a Chlamydia trachomatis (chlamydia) vaccine but research to achieve this continues. It is therefore important to evaluate the potential impact of different chlamydia vaccine strategies on population prevalence. The optimal strategy might differ depending on whether a vaccine prevents chlamydia infection, or prevents ascending infection to reduce the risk of long term complications. Here, we used a mathematical model to focus on vaccination strategies that aim to reduce population prevalence.

Methods We developed a deterministic pair model of heterosexuals aged 15-39 years that incorporates the formation and dissolution of sexual partnerships. We used sexual behavioural data from UK population-based studies to inform the model. The model has a baseline chlamydia prevalence of $3 \%$. In all strategies examined, vaccination was introduced before sexual debut. We investigated the impact on population prevalence of different types of vaccine coverage, vaccine efficacy and duration of protection. We also assumed different types of protection after vaccination. We started with full protection, but since animal models have shown that sterilising immunity is difficult to achieve we also investigated partial protection of the vaccine by assuming a decreased susceptibility to infection after vaccination or, when infected, a shorter duration of infection or a reduced transmission probability. 